\title{
NOTICIAS
}

\section{Sugestión de la Sociedad Mexicana de Salud Pública sobre el control de la adicción a la nicotina}

En algunos países industrializados, las estrategias integrales antitabaco -que incluye la legislación para el control del tabaco, políticas de precios y educación para la salud- han sido efectivas para reducir el número de fumadores. Los países que han introducido regulación de precios y han hecho disponible al público la información sobre los riesgos de fumar tabaco voluntaria o pasivamente han creado un clima social y político más receptivo para sentar las bases jurídicas para el control de la adicción a la nicotina. Se ha probado que los programas de prevención y de educación son exitosos y costo-efectivos y que el aumento en los impuestos al tabaco reduce el consumo y produce mayores ingresos al gobierno. Algunos estudios han mostrado que un incremento de $10 \%$ en el precio de los cigarros reduce su consumo en $2-5 \%$ en adultos y hasta $10 \%$ en los jóvenes y los desanima para empezar a fumar. ${ }^{1,2}$
Sin embargo, el control de los precios y del mercado utilizado con éxito en algunos países industrializados, no se ha aplicado a los productos de tabaco exportados. Los países industrialmente desarrollados no han compartido con los países en desarrollo suficiente información acerca de las estrategias exitosas para la operacionalización de los programas de prevención y control. Muchos ciudadanos de los países en desarrollo aún ignoran la información básica acerca de los efectos dañinos que el tabaco tiene contra la salud.

Para las compañías transnacionales, México representa un mercado potencial altamente rentable por las siguientes razones: a) la industria del tabaco procura y estimula el mercado de los jóvenes (30 millones entre 10 y 24 años de edad), quienes son los clientes más susceptibles para desarrollar la adicción a la nicotina para el resto de su vida, y b) la economía en México está en franco ascenso, lo que se traduce en que los jóvenes tengan los recursos financieros personales necesarios para comenzar a fumar tabaco.
Actualmente, los recursos del sector salud para prevenir y combatir esta adicción son insuficientes para competir con la promoción que hacen las compañías tabacaleras internacionales. El Plan Nacional Antitabaco permanece sin financiamiento en comparación con el presupuesto utilizado por las tabacaleras para su propaganda. En 1996, se estimó que la inversión de las compañías tabacaleras era de más de 3 billones de pesos solamente en publicidad. Estas cifras contrastan con los magros recursos que se usan actualmente en actividades de prevención; por ejemplo, el Consejo Nacional contra las Adicciones cuenta con un presupuesto que no alcanza un millón de pesos, cantidad claramente insuficiente.

La indisputable evidencia científica indica que fumar tabaco incrementa la frecuencia de la enfermedad obstructiva crónica del pulmón, enfisema, cáncer del pulmón y enfermedad cardiaca. En México -país en la etapa inicial de la epidemia mundial del tabacose estiman cada año 6000 muertes de cáncer del pulmón y 8000 por

La Sociedad Mexicana de Salud Pública está integrada por sociedades estatales y sanitaristas multidisciplinarios nacionales cuyos miembros han sido profesionales interesados en la salud pública. Fundada en 1944, la Sociedad Mexicana de Salud Pública es una de las más antiguas asociaciones de este tipo en la región de las Américas. Como asociación civil es un foro independiente en el que se discuten los factores determinantes de los daños a la salud colectiva de los ciudadanos, propone soluciones a los problemas que afectan tanto las funciones de los seres humanos como la integridad y sanidad del ambiente. Además, brinda cooperación técnica para el desarrollo de infraestructuras, programas, políticas y estrategias de salud pública, de investigaciones especiales y de formación de recursos humanos en salud colectiva. 
enfermedades cardiovasculares debidas a esta adicción. En 1997, se estimó en alrededor de 14 millones de fumadores de tabaco en el país, los cuales tenían en promedio 30 años de edad. En 1995, había 4 millones de fumadores jóvenes y cada año se suman 100000 nuevos adictos.

Conforme bajan las ventas en los países desarrollados, la industria tabacalera busca nuevos mercados en poblaciones más vulnerables de los países en desarrollo. Consecuentemente, podría haber un aumento catastrófico en las tasas de fumadores. En México, el consumo de cigarros se ha mantenido entre 2500 y 3000 millones de cajetillas de éstos al año durante el periodo 1980-1996. Para el año 2010 se acumularán 123000 muertes por cáncer del pulmón y 198000 por enfermedad isquémica del corazón, atribuibles al consumo de tabaco.

Los escasos recursos financieros en los países en desarrollo hacen casi imposible para los gobiernos y las autoridades de salud pública hacer un "ataque frontal" contra las estrategias multimillonarias de propaganda de las compañías tabacaleras. La presión impuesta por procesos de transición política, económica, demográfica y epidemiológica velan la posibilidad de que el control del tabaquismo sea prioridad en la agenda de la política pública del poder ejecutivo. Como resultado, el alerta público sobre las repercusiones del uso del tabaco en la salud es muy bajo o inexistente en la mayoría de los países en desarrollo.

\section{Sugestión}

La Sociedad Mexicana de Salud Pública sugiere al secretario de Salud la adopción de las políticas y estrategias convenidas en el ConvenioMarco sobre la Lucha Antitabáquica (CMLA) de la Organización Mun- dial de la Salud (OMS), adaptadas a la realidad del país. También sugiere tomar en consideración los siguientes argumentos estratégicos que podrían servir de base para tomar la decisión política que identifique al control del tabaco como acción prioritaria, y dé respuesta a la epidemia del tabaquismo mundial.

\section{La participación de México en la} agenda global para el control del tabaco

En el pasado reciente, México ha intervenido en diversos talleres, reuniones y proyectos en los que se ha llegado a acuerdos, resoluciones y propuestas de los más altos niveles de toma de decisiones, encaminados a la construcción de una agenda para el control del tabaco. ${ }^{3-19}$

Las medidas de control efectivo del tabaco pueden ser operacionalizadas solamente si se crea un ambiente de apoyo social, político y financiero con bases jurídicas sólidas. Esto requiere una perspectiva completamente nueva en relación con el tabaco: la nicotina es una droga adictiva y su control es un problema político.

La estrategia para el control de la nicotina debe ser holística, con el fin de involucrar no sólo a los sectores sociales de salud y educación sino a todas los instituciones del gobierno, a todas las profesiones relacionadas con el desarrollo integral del hombre y a toda la población del país. Mientras que algunos aspectos de la política para el control del tabaco son muy efectivamente tratados en los ámbitos local, estatal y nacional, otros aspectos demandan atención y colaboración regional y global.

En México se ha decidido incluir en la agenda política de prioridades la protección de la salud de los niños y de los adolescentes. Los propósitos inmediatos son:
- evitar que los menores de edad inicien el hábito de fumar tabaco;

- reducir/eliminar el consumo de tabaco por niños y jóvenes;

- prevenir el tabaquismo involuntario en niños y jóvenes, y

- prevenir el tabaquismo activo y pasivo en las mujeres grávidas.

Actividades sustantivas como la educación para la salud se pueden iniciar de inmediato, mientras que otras medidas más complejas y de difícil operacionalización requieren esfuerzo a largo plazo. Para impulsar estrategias antitabaco efectivas es necesario enfocar tres áreas principales:

- legislación para el control del tabaco;

- políticas de precio, comercialización y mercado, y

- educación para la salud en relación con el hábito de fumar y la adicción a la nicotina.

\section{Prevención, control y tratamiento}

La educación para la salud es un requisito sine qua non para promover la señal de alerta sobre las consecuencias de fumar tabaco. Mientras la educación para la salud da poder a los individuos, la legislación para el control del tabaco da poder a los países, lo que permite a los gobiernos reforzar los programas antitabáquicos.

México, como país protagónico en el concierto de la salud pública internacional, puede dar los siguientes pasos para ayudar a alcanzar un mundo libre de tabaco en los siguientes rubros:

\section{Legislación para el control del tabaco}

- unirse a las autoridades de los gobiernos del mundo para regular la nicotina como una droga 
adictiva y a los cigarros y otros productos de tabaco como instrumentos de administración de la droga;

- participar en la adopción de una legislación para actividades de colaboración para el control global del tabaco por todos los países del mundo;

- estimular la prohibición de la ventas de tabaco a los menores de edad por medio del cumplimiento estricto de la ley y la aplicación de mecanismos de seguimiento;

- urgir la prohibición de toda propaganda, promoción y auspicio de eventos laborales, escolares, deportivos, culturales, recreativos y artísticos con tabaco, directa o indirectamente, en todos los medios;

- facilitar la comunicación entre los sectores de salud y otros sectores del gobierno y de la sociedad (inclusive los sectores de economía, educación, vivienda, transporte, cultura, justicia, industria y comercio, agricultura, trabajo y ambiente) para construir el apoyo para una legislación efectiva antitabáquica;

- desarrollar e implantar restricciones a la venta y uso del tabaco en lugares públicos, para proteger a todos los seres humanos de la exposición involuntaria al humo del tabaco en el ambiente, estimulando el uso de avisos prohibitivos y la creación y ampliación de áreas libres de humo de tabaco en sitios públicos, hoteles y restoranes;

- asegurar que se requieran etiquetas sobre daños a la salud, colocadas prominentemente en los paquetes de cigarros en el lenguaje local, y en todos los productos de tabaco por igual, en todo el mundo, $\mathrm{y}$

- asegurar que los derechos legales de la gente estén comple- tamente protegidos aunque no sean parte de cualquier convenio nacional.

\section{Políticas de precio y mercadeo}

- unirse a todos los países para prohibir a todas las autoridades gubernamentales que promuevan ventas nacionales de tabaco y a exportarlo, interfiriendo con el esfuerzo de las autoridades internacionales $\mathrm{o}$ extranjeras de salud para regular el tabaco, y desalentar leyes débiles para el control del tabaco en cualquier país;

- abogar por: a) la armonización internacional de los precios e impuestos del tabaco a niveles altos; b) asignación de los fondos adquiridos para financiar las medidas de control del tabaco, el tratamiento de las personas adictas y de las que sufren enfermedades relacionadas con el tabaco; c) el desarrollo de alternativas económicas para sustituir el cultivo y manufactura de productos de tabaco, y d) prohibir el uso de tecnologías que produzcan plantas de tabaco con mayor contenido de nicotina, así como el uso de plaguicidas (altamente tóxicos para el hombre) en las plantaciones de tabaco;

- abogar porque las compañías tabacaleras se responsabilicen por proveer fondos suficientes para el diagnóstico y tratamiento adecuado de personas adictas y de las que sufren de enfermedades producidas como consecuencia de la exposición al humo de tabaco en el ambiente;

- abogar para que se excluyan convenios de libre comercio del tabaco en la Organización Mundial de Comercio, con el objeto de permitir que los países regulen a las compañias multinacionales de tabaco, sin miedo de sanciones por prácticas injustas de comercio;

- abogar para salvaguardar en contra del contrabando internacional de tabaco por medio de un sistema de permisos de exportación, mejoría del control del registro e informes de la producción y ventas, penalizaciones estrictas y otras medidas;

- urgir la terminación de las ventas de tabaco y sus productos libres de impuestos en todo el mundo, $\mathrm{y}$

- desalentar a los gobiernos para que acepten nuevas inversiones para las compañías tabacaleras transnacionales y obstaculizar la compra de compañías nacionales en bancarrota o en decadencia.

\section{Educación para la salud, control} del tabaco y tratamiento de adictos a la nicotina

- apoyar el desarrollo y operacionalización de programas y campañas formales de educación antitabaco tanto en las escuelas como fuera de ellas, comenzar en el nivel prescolar;

- estimular el reconocimiento y la certificación de instituciones docentes libres de nicotina;

- promover el alerta público sobre los daños de fumar a través de los medios masivos de comunicación;

- elaborar materiales de educación para la salud apropiados a las culturas y edades, y hacerlos profusamente disponibles para el público;

- apoyar el adiestramiento de todos los proveedores de salud (inclusive los administradores, antropólogos, abogados, biólogos, bioestadísticos, cirujanos, contadores, ecólogos, economistas, enfermeros, entomólogos, 
farmacéuticos, gerentes, ginecoobstetras, ingenieros, laboratoristas, legisladores, nutriólogos, médicos, odontólogos, pediatras, psicólogos, trabajadores comunitarios de salud y veterinarios), en la prevención y tratamiento del hábito de fumar y la adicción a la nicotina.

- urgir a los empleados de todas las dependencias oficiales y de todas las empresas e industrias públicas y privadas a promover el ambiente de trabajo libre de humo de tabaco;

- dar el ejemplo, esto es, estar totalmente libres del humo del tabaco en los ambientes familiar, laboral, escolar, cultural y recreativo (lo que incluye cines y teatros), así como en transportes públicos terrestres y aéreos y centros comerciales de autoservicio;

- estimular el desarrollo del tratamiento farmacológico eficaz para la adicción a la nicotina y otros métodos para dejar de fumar tabaco;

- trabajar para movilizar los recursos financieros para un programa de investigación antitabáquico y para desarrollar y operacionalizar el programa antitabáquico eficaz y permanente, $y$

- alentar a las autoridades para que una parte importante de la inversión en propaganda de las compañías tabacaleras se asigne a la investigación de los daños ocasionados por el tabaco, al diagnóstico y tratamiento oportuno de las enfermedades ocasionadas por el tabaco y a la recuperación de las personas adictas a la nicotina.

\section{Fortalecimiento institucional.El compromiso del sectorsalud}

El Instituto Nacional de Salud Pública (INSP) ha decidido crear el Cen- tro de Investigaciónes y de Acción Antitabaco, cuyos objetivos principales son generar y diseminar conocimiento relacionado con la evolución de la epidemia mundial de adicción a la nicotina y formar recursos humanos capaces de ejecutar acciones efectivas para reducir/ eliminar el uso del tabaco. Asimismo, el centro estima los costos de la atención médica resultante de fumar tabaco; promueve políticas de impuesto al valor agregado para apoyar actividades antitabaco y para el tratamiento de enfermedades ocasionadas por el humo de tabaco en el ambiente y desarrolla sistemas de información y diseminación en un centro de internet sobre las actividades de control y sus resultados, dirigidas a grupos sociales especiales, como niños, mujeres, adolescentes y maestros.

La asociación entre el Global Tobacco Institute y el INSP satisface la urgente necesidad de desarrollar un centro que se utilice como recurso para las actividades antitabáquicas y que asegure la rápida transferencia del conocimiento adquirido en los Estados Unidos de América hacia México y la apropiada traducción y adaptación de ese conocimiento a la sociedad mexicana. Además, la influencia que el INSP tiene en países latinoamericanos se puede aprovechar para reproducir sus actitividades en los países de la región que enfrentan problemas semejantes. Esta articulación promoverá la cooperación técnica entre países latinoamericanos con beneficios mutuos.

Se necesita financiamiento para desarrollar el centro de información y el currículo para cursos antitabaco. Las actividades de este centro de información se pueden operacionalizar de inmediato en virtud de que se han identificado varias bases de datos inexploradas que se pueden convertir en información valiosa relativa al uso del tabaco. Las actividades a desarrollar por el cen- tro incluyen recopilación, documentación, análisis y distribución de las siguientes bases de datos: encuestas nacionales sobre diferentes tópicos conducidas en los últimos 12 años, las cuales tienen informaciones sobre fumar tabaco; encuesta nacional sobre ingresos y gastos (inclusive en tabaco), por familia conducidas cada cuatro años; exámen de admisión de estudiantes a la Universidad Nacional Autónoma de México, desde 1980 (50 000 por año), el cual indaga sobre prevalencia de fumar tabaco.

Los cursos antitabaco están dirigidos a todas las personas involucradas en los programas del Control de la Adicción a la Nicotina y actualizará a los participantes en las siguientes áreas: epidemiología del uso del tabaco y de la adicción a la nicotina, efectos en la salud, tratamiento médico de la adiccción, estrategias de prevención, teoría de los cambios de comportamiento, utilización de la población blanco, economía de la adicción, la industria del tabaco, impuestos sobre el tabaco, legislación para proteger no fumadores, diseño, gerencia y evaluación de programas nacionales de control y papel de las organizaciones no gubernamentales en el control de la epidemia.

\section{El compromiso dela Federación Mundial de Asociaciones deSalud Pública}

El reto para conseguir un mundo libre de tabaco demanda un esfuerzo comprehensivo y de colaboración en el ámbito global. En 1996, la 49a. Asamblea Mundial de la Salud pidió a la Dirección General de la OMS desarrollar una convención y marco de referencia internacional para el control del tabaco. Este podría ser en forma de tratado internacional por medio del cual los Estados signatarios estarían de acuerdo en conseguir metas definidas para el control 
del tabaco. El marco de referencia aborda problemas como propaganda, mercadeo, ventas internacionales, precios e impuestos, contrabando e intercambio de información sobre políticas y programas.

La Sociedad Méxicana de Salud Pública, como miembro de la World Federation of Public Health Associations (WFPHA), reconoce que en México tenemos la oportunidad de aniquilar uno de los mayores flagelos de la salud pública en este milenio. Como miembros de la WFPHA comprometemos nuestros recursos y energía para ayudar a desarrollar e implementar la convención marco de referencia internacional de la OMS y otros esfuerzos globales contra el tabaco. Asimismo, sugerimos a las autoridades de salud de México demandar la regulación en el ámbito mundial de la nicotina como droga psicoactiva y adictiva.

Colofón: trabajemos activamente con los gobiernos nacionales y estatales, agencias intergubernamentales, organizaciones no gobernamentales, negocios y grupos de influencia para promover un mundo libre de tabaco. ${ }^{20}$

Francisco Javier López Antuñano, Director de Relaciones Internacionales,

Sociedad Mexicana de Salud Pública, México.

Correo electrónico: alantu@ insp3.insp.mx

\section{REFEREN CIAS Y DOCUMEN TOS CONSULtAdOS}

1. W orld Health 0 rganization. Tobacco epidemic: Much more than a health issue. Fact Sheet $\mathrm{N}$ o. 155, May 1997.

2. Warner K, E. Tobacco taxation as health policy in the Third W orld. Am J Public Health 1990;80(5). 3. O rganización Panamericana de la Salud. El Convenio Marco sobre la Lucha Antitabática. Una primicia. El CMLA no es un convenio ordinario, es en potencia un movimiento de salud pública. O PS,1999. D ocumentos no publicados. 4. Organización Panamericana de la Salud. XLI Consejo directivo O PS/O MS. Tobacco control in the Americas. Punto 4.2 CD 41/7 7 julio 1999 5. Organización Panamericana de la Salud. Movilización para el Convenio Marco sobre la Lucha Antitabáquica (CMLA) Resumen de la discusión, Taller para la prevención y control del tabaquismo. O PS/O MS, Diciembre, 1999.

6. Instituto N acional de Salud Pública. El tabaquismo en México. Acciones urgentes para prevenir una epidemia. Propuesta. IN SP/C ISP, 1997. 7. Proposal for the G lobal Tobacco Institute (GTI, Baltimore, $\mathrm{Md}$ ), and the $\mathrm{N}$ ational Institute of Public Health mutual agreement for the devopment of the Anti-Tobacco Research and Action Center (ATRAC, Cuernavaca, Morelos), 2000.

8. Prevention and control of tobacco use. Draft. Regional Proposal HPP/PAHO 2000-2001.

9. O rganización Mundial de la Salud. Convenio Marco sobre la Lucha Antitabáquica de la O MS. Informe de la primera reunión del Grupo de Trabajo. O MS, 25-29 de octubre de 1999.

10. Aspectos económicos de la lucha antitabáquica. Primera reunión del grupo de trabajo sobre el Convenio Marco sobre la Lucha Antitabáquica de la O MS. O PS/O MS, 1999.
11. W orld Health O rganization. Confronting the Epidemic. A global agenda for tobacco control research. Ginebra: W HO , July 1999.

12. Secretaría de Salud. Subsecretaría de Prevención y Control de Enfermedades. Consejo $\mathrm{N}$ acional Contra las Adicciones. Programa de Prevención y Control del Tabaquismo. 1998-2000. México, D.F.: SSA.

13. Estados Unidos Mexicanos. Presidencia de la República. Proyecto de Reglamento de la Ley General de Salud en Materia de Publicidad. 2000. Documento no publicado.

14. O rganización Panamericana de la Salud. Prevención y control del tabaquismo. Proyecto Interagencial Tabaco o Salud en Latinoamérica. OPS, 1999. Documento no publicado.

15. Plan de acción para operacionalizar el Programa Interagencial Tabaco o Salud de CLACCTA/ O PS. Proyecto Interagencial Tabaco o Salud en Latinoamérica. Propuesta, 1999. D ocumento no publicado.

16. Development in Practice. Curbing the epidemic. Governments and the Economics of Tabacco Control. 1999 The International Bank for Reconstruction and Development/ The World Bank. W ashington, DC.

17. May 31, 1999 "W orld N oTobacco Day" Leave the pack behind. Statement by Dr. George A.O. Alleyne, Director, Pan American Health O rganization. www.paho.org

18. General information for the establishment of a sustainable system of epidemiological tracking and follow-up. Unpublished Document. W ashington, D.C.: O PS/O MS.

19. Grupo Catalizador. Cruzada N acional Que no fumen las chavas ni los chavos. Metas del Programa de Reforma del Sector Salud, SSA, 1999. Do cumento no publicado.

20. World Federation of Public Health Association. Position Paper on Tobacco. N ovember, 1999. 